\title{
ELIMINATION OF FIRST ORDER ERRORS IN SHOCK CALCULATIONS*
}

\author{
GUNILLA KREISS ${ }^{\dagger}$, GUNILLA EFRAIMSSON ${ }^{\ddagger}$, AND JAN NORDSTRÖM $\ddagger \S$
}

\begin{abstract}
First order errors downstream of shocks have been detected in computations with higher order shock capturing schemes in one and two dimensions. Based on a matched asymptotic expansion analysis we show how to modify the artificial viscosity and raise the order of accuracy.
\end{abstract}

Key words. hyperbolic conservation law, artificial viscosity, central difference scheme, asymptotic analysis

AMS subject classifications. 35L65, 35L67, 65M06, 65M12

PII. S0036142998349412

1. Introduction. Phenomena that require very high resolution, i.e., high order finite difference methods, occur in applications such as electromagnetics, acoustics (in fact, all cases of wave propagation), and direct simulation of turbulent and transitional flow (see, for example, [3], [6], [14], [15], [16], [17], [18], [19], [20]). The small scale behavior is of significance in the applications above, and the phenomena involved cannot be captured without an appropriate resolution of this fine scale. The efficiency [10] of high order finite difference methods compared to low order methods can, of course, also be used to reduce the computational cost for a given level of accuracy.

However, recently it has been reported (see [1], [2], [5]) that solutions of conservation laws obtained by formally high order accurate schemes degenerate to first order downstream of a shock layer. The effect is seen only when (i) the characteristics come out of the shock region and (ii) the solution is nonconstant. Examples in one space dimension where both these conditions are satisfied include steady state calculations for systems with a source term and time dependent calculations for systems with nonconstant initial data.

The downstream degeneration of accuracy has also been observed in calculations of acoustic waves in the presence of shocks. This case can be modeled by a scalar, linear equation where the wave speed changes value in a thin region without changing sign (see [2] and references therein). The degeneracy in accuracy is troublesome, even though the first order terms for reasonable mesh-sizes seem to be small in many cases.

In [4], an explanation of the degeneracy in accuracy on solutions of conservation laws were given. Steady state solutions to systems with source terms were analyzed by using matched asymptotic expansions for a corresponding viscous equation, the so-called modified equation; see [13]. It was assumed that an inner and an outer solution exist. The inner solution is valid in the shock region and the outer solution elsewhere. The two solutions are matched in a so-called matching zone. The inner solution, the outer solution, and the shock position are expanded in powers of the small viscosity coefficient $\varepsilon$. With $\varepsilon=\mathcal{O}(h)$ in the shock region, the outer solution contains a term of $\mathcal{O}(h)$ downstream of the shock. Upstream this term is zero.

\footnotetext{
* Received by the editor December 17, 1998; accepted for publication (in revised form) September 28, 2000; published electronically January 25, 2001.

http://www.siam.org/journals/sinum/38-6/34941.html

†NADA, KTH, SE-100 44 Stockholm, Sweden (gunillak@nada.kth.se).

${ }^{\ddagger}$ FFA, Box 11021, SE-161 11 Bromma, Sweden (eng@ffa.se, nmj@ffa.se).

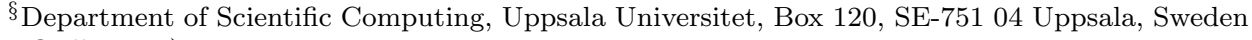
(nmj@tdb.uu.se).
} 
In the present paper we also consider steady state solutions of systems with source terms. We use the same asymptotic technique as in [4], and find that with a specific choice of a matrix valued artificial viscosity the downstream $\mathcal{O}(h)$-error is eliminated. With this artificial viscosity we construct a numerical scheme for a specific system. We present calculations that are second order accurate both upstream and downstream of the shock region. The analysis and the computations show that it is possible to construct truly higher order methods.

2. Hyperbolic system of conservation laws with a source term. We consider

$$
\mathbf{v}_{t}+\mathbf{f}(\mathbf{v})_{x}+\alpha(x) \mathbf{v}=0, \quad 0 \leq x \leq 1,
$$

where $\mathbf{v}=\left(v^{(1)}, v^{(2)}, \ldots, v^{(n)}\right)^{T}$ and $\alpha(x)$ is a smoothly varying function. Equation (2.1) can be viewed as a simplified form of the nozzle equations, where the lower order term is nonlinear, or the equations in geophysical fluid dynamics where the Coriolis forces enter as linear lower order terms.

We denote the Jacobian of the flux function by $J$. The eigenvalues of $J\left(\lambda_{i}, i=\right.$ $1,2, \ldots, n)$ are purely real. Without loss of generality we order them in increasing order. If $x_{s}$ is a point of discontinuity traveling with speed $s$, we require the solution to satisfy the Rankine-Hugoniot condition

$$
s[\mathbf{v}]=[\mathbf{f}] \quad \text { at } x=x_{s} .
$$

Here $[\mathbf{v}]=\mathbf{v}_{+}-\mathbf{v}_{-}$, where $\mathbf{v}_{+}=\lim _{\delta \rightarrow 0+} \mathbf{v}\left(x_{s}+\delta\right)$ and $\mathbf{v}_{-}=\lim _{\delta \rightarrow 0+} \mathbf{v}\left(x_{s}-\delta\right)$. We call the discontinuity a $k$-shock if it in addition satisfies the Lax entropy condition $[12]$

$$
\begin{aligned}
& \lambda_{k-1}^{-}<s<\lambda_{k}^{-}, \\
& \lambda_{k}^{+}<s<\lambda_{k+1}^{+},
\end{aligned}
$$

where $\left.\lambda_{k}^{ \pm}=\lambda_{k}\left(v_{ \pm}\right)\right)$. This means that exactly $n+1$ characteristics impinge on the shock.

In this paper we will consider a so-called 1-shock. If the eigenvalues are of constant sign in smooth regions, then suitable boundary conditions are (see, e.g., [9])

$$
\begin{array}{ll}
\mathbf{v}=\mathbf{v}_{0} & \text { at } x=0, \\
S(\mathbf{v})=r & \text { at } x=1,
\end{array}
$$

where $\mathbf{v}_{\mathbf{0}} \in \mathrm{R}^{n}$ and $r \in \mathrm{R}$ are given and $S(\mathbf{v}): \mathrm{R}^{n} \rightarrow \mathrm{R}$.

We make the following assumption.

Assumption 2.1. There exists a steady, piecewise smooth solution of (2.1) and (2.3) with a 1-shock at $x_{s}$. Also, the eigenvalues are nonzero in the smooth parts of the solution, i.e., $\lambda_{i}>0 \forall i$ for $0 \leq x<x_{s}$ and $\lambda_{1}<0$ and $\lambda_{i}>0 i \neq 1$ for $x_{s}<x \leq 1$.

Many numerical solutions of (2.1) can be viewed as higher order accurate solutions of the slightly viscous, so-called modified equations

$$
\mathbf{u}_{t}+\mathbf{f}(\mathbf{u})_{x}+\alpha(x) \mathbf{u}=\left(\Gamma \mathbf{u}_{x}\right)_{x}
$$

(see, e.g., [13]). The viscous terms damp oscillations in the numerical solution. In the neighborhood of a shock layer the matrix $\Gamma=\mathcal{O}(h)$, where $h$ is the grid size. That 
is, $|\Gamma / h|<c$ as $h \rightarrow 0$ and $c$ is a constant. In regions where the solution is smooth the viscosity term can be of $\mathcal{O}\left(h^{2}\right)$ or smaller. A so-called limiter determines when to switch on the larger viscous terms. There is a wide variety of limiters and comprehensive work has been performed in order to analyze theoretically and practically the behavior of different limiters. In this article, we will not go into the details of how to construct limiters.

More terms can be included in the modified equation. For instance, with a first or second order accurate approximation of the flux derivative, a dispersive term $\left(\Gamma_{2} \mathbf{v}_{x x}\right)_{x}$ with $\Gamma_{2}=\mathcal{O}\left(h^{2}\right)$ is present; see [13]. In the region away from the shock, higher order terms will not affect the analysis. In the shock region, however, higher order terms may be of importance except in the case of weak shocks (see the discussion in [7]).

In this paper we consider

$$
\mathbf{f}(\mathbf{u})_{x}+\alpha(x) \mathbf{u}=\varepsilon^{2} \mathbf{u}_{x x}+\varepsilon\left(\phi(x) E(\mathbf{u}) \mathbf{u}_{x}\right)_{x}
$$

with $\varepsilon=O(h)$. That is, $\Gamma=\varepsilon^{2} I+\phi \varepsilon E$. Here $\phi$ is a smooth function of $x / \varepsilon$ satisfying

$$
\phi(x)= \begin{cases}1 & \text { for }\left|x-x_{s}\right| \leq \varepsilon K \\ 0 & \text { for }\left|x-x_{s}\right| \geq \varepsilon(K+1) .\end{cases}
$$

Here $x_{s}$ is the position of the inviscid shock, and $K$ is a sufficiently large constant.

We consider the same boundary conditions as for the inviscid problem (2.3) augmented with $n-1$ gradient boundary conditions at $x=1$. The gradient conditions guarantee that at most a weak boundary layer is formed at the outflow boundary.

In this paper we will use matched asymptotic expansions. We make the following assumption.

Assumption 2.2. The solution of (2.5) can be described by an inner solution, valid in the shock layer, and an outer solution, valid elsewhere. The inner solution is a function of the stretched variable $\tilde{x}=\left(x-x_{s}\right) / \varepsilon$. These solutions can be expanded in $\varepsilon$ and matched to sufficient order in a region of overlap. Further, the position of the shock layer can also be expanded in $\varepsilon$. Thus we have expansions on the form

$$
\begin{array}{ll}
\text { Inner: } & \mathbf{u} \sim \mathbf{g}_{0}(\tilde{x})+\varepsilon \mathbf{g}_{1}(\tilde{x})+\eta_{2}(\varepsilon) \mathbf{g}_{2}(\tilde{x})+\ldots \\
\text { Outer: } & \mathbf{u} \sim \mathbf{w}_{0}(x)+\varepsilon \mathbf{w}_{1}(x)+\gamma_{2}(\varepsilon) \mathbf{w}_{2}(x)+\ldots \\
\text { Position: } & x_{\varepsilon} \sim x_{0}+\varepsilon x_{1}+\sigma_{2}(\varepsilon) x_{2}+\ldots
\end{array}
$$

Here $\mathbf{w}_{0}=\mathbf{v}$ and $x_{0}=x_{s}$. Also, $\eta_{2}(\varepsilon)=\boldsymbol{o}(\varepsilon), \gamma_{2}(\varepsilon)=\boldsymbol{o}(\varepsilon)$, and $\sigma_{2}(\varepsilon)=\boldsymbol{o}(\varepsilon)$; that is, $\lim _{\varepsilon \rightarrow 0}\left|\eta_{2}(\varepsilon) / \varepsilon\right|=0$ and accordingly for $\gamma_{2}$ and $\sigma_{2}$. We denote the position of the viscous shock layer as the point where the smallest eigenvalue of the Jacobian, evaluated along the viscous solution, changes sign. The matching can be done at points $x_{m}^{-}$and $x_{m}^{+}$satisfying $x_{0}-M \leq x_{m}^{-} \leq x_{0}-m$ and $x_{0}+m \leq x_{m}^{+} \leq x_{0}+M$, respectively, where $\varepsilon \ll m<M \ll 1$ and $M \rightarrow 0$ as $\varepsilon \rightarrow 0$.

Note that the large viscosity is not present in the matching region.

In [4] we derived equations for $\mathbf{w}_{1}$ and $x_{1}$ in the case of a viscosity matrix $E=I$, using standard matched asymptotic techniques; see [8] or [11]. Now we will derive similar equations with a more general matrix $E$.

By introducing the inner expansion into (2.5) and making a change of variables, $\tilde{x}=\left(x-x_{s}\right) / \varepsilon$, we find to zeroth order in $\varepsilon$ that

$$
\mathbf{f}\left(\mathbf{g}_{0}\right)_{\tilde{x}}=\left(\phi(\tilde{x}) E\left(\mathbf{g}_{0}\right) \mathbf{g}_{0 \tilde{x}}\right) \tilde{x} .
$$


In accordance with standard asymptotic techniques, [8], [11], we use the inviscid solution at the shock position $x=x_{s}$ as boundary data at $\tilde{x}= \pm \infty$. If $g_{0}$ is sufficiently close to the boundary values at $\tilde{x}= \pm K$, where $K$ is defined in (2.6), then we can replace $(2.8)$ by

$$
\mathbf{f}\left(\mathbf{g}_{0}\right)_{\tilde{x}}=\left(E\left(\mathbf{g}_{\mathbf{0}}\right) \mathbf{g}_{0 \tilde{x}}\right)_{\tilde{x}}
$$

Clearly the above equation cannot have a unique solution. If $g_{0}(\tilde{x})$ is a solution, then so is $g_{0}(\tilde{x}+c)$. Let $\mathbf{g}(\tilde{x})$ satisfy

$$
\mathbf{f}(\mathbf{g})_{\tilde{x}}=\left(E(\mathbf{g}) \mathbf{g}_{\tilde{x}}\right)_{\tilde{x}},
$$

together with the additional condition that the smallest eigenvalue of $J(\mathbf{g})$ vanishes at $\tilde{x}=0$. Here $J$ is the Jacobian of the flux function $\mathbf{f}$. Then $\mathbf{g}_{0}(\tilde{x})=\mathbf{g}\left(\tilde{x}-x_{1}\right)$, where $x_{1}$ is the first order perturbation of the position of the shock layer. Below we will determine $x_{1}$ by considering the outer problem to $\mathcal{O}(\varepsilon)$.

Away from the shock layer, the outer expansion introduced into (2.5) yields to zeroth order the inviscid problem. To first order we have

$$
\left(J(\mathbf{v}) \mathbf{w}_{1}\right)_{x}+\alpha(x) \mathbf{w}_{1}=0 .
$$

From the boundary conditions at $x=0$ and $x=1$ we have

$$
\begin{array}{r}
\mathbf{w}_{1}(0)=0, \\
\mathbf{L}^{T} \mathbf{w}_{1}(1)=0,
\end{array}
$$

where $\mathbf{L}^{T}=\frac{\partial S}{\partial v}\left(\mathbf{w}_{0}\right)$. It follows that $\mathbf{w}_{1} \equiv 0$ in the upstream region. This is in agreement with [5], where it is proved that in the corresponding discrete case, $\mathbf{w}_{1}$ is not present in the upstream region when the numerical scheme is linearly stable and contractive. Consequently, we need only to consider (2.11) in the downstream region $x_{m}^{+} \leq x \leq 1$. However, a boundary condition at $x=x_{m}^{+}$is thus required. By integrating (2.5) from matching point to matching point one obtains to lowest order the Rankine-Hugoniot condition. By including terms to the next order and comparing with the correspondingly integrated inviscid equation we obtain the boundary condition at $x=x_{m}^{+}$

$$
J^{+} \mathbf{w}_{1}\left(x_{m}^{+}\right)+x_{1} \alpha\left(x_{s}\right)[\mathbf{v}]=-\mathbf{I}
$$

where

$$
\mathbf{I}:=\int_{-\infty}^{0} \alpha\left(x_{s}\right)\left(\mathbf{g}(\tilde{x})-\mathbf{v}_{-}\right) d \tilde{x}+\int_{0}^{\infty} \alpha\left(x_{s}\right)\left(\mathbf{g}(\tilde{x})-\mathbf{v}_{+}\right) d \tilde{x} .
$$

A detailed derivation of (2.14) can be found in Appendix A.

For future reference we write out the equation and boundary conditions for $\mathbf{w}_{1}$ for $x_{m}^{+} \leq x \leq 1$ :

$$
\begin{array}{r}
\left(J(\mathbf{v}) \mathbf{w}_{1}\right)_{x}+\alpha(x) \mathbf{w}_{1}=0, \\
J^{+} \mathbf{w}_{1}\left(x_{m}^{+}\right)+x_{1} \alpha\left(x_{s}\right)[\mathbf{v}]=-\mathbf{I}, \\
\mathbf{L}^{T} \mathbf{w}_{1}(1)=0,
\end{array}
$$

where $\mathbf{I}$ is defined in (2.14) and $\mathbf{L}^{T}=\frac{\partial S}{\partial v}\left(\mathbf{w}_{0}\right)$. In general $\mathbf{w}_{1}$ is nonzero. This is confirmed by computations presented in [4]. 
It is possible to derive equations for terms up to any order in $\varepsilon$. The equations will all be linear and of the same type. Assumption 2.2 implies that the linear operators involved have reasonable properties (uniqueness, existence, continuous dependence on forcing, etc). In particular the problem (2.15) is well posed when Assumption 2.2 is valid.

Remark 2.1. The case of a $k$-shock, $k>1$ can be treated analogously. Instead of (2.15) one obtains an equation for $\mathbf{w}_{1}$ on both sides of the shock, coupled with a linearized jump condition with the same forcing as above.

We will now address the question of how to ensure $\mathbf{w}_{1} \equiv 0$ in the downstream region. That (2.15) is well posed implies that $\mathbf{w}_{1}$ and $x_{1}$ are uniquely determined by the right-hand side $\mathbf{- I}$. There are two possibilities. If $\mathbf{I}=0$, the unique solution of (2.15) is $\mathbf{w}_{1} \equiv 0$ and $x_{1}=0$. To avoid imposing the symmetry requirements on the shock profile implied by $\mathbf{I}=0$, we have pursued another possibility. If we require $\mathbf{I}$ to be parallel to $[\mathbf{v}]$, then $(2.15)$ is satisfied by $\mathbf{w}_{1} \equiv 0, x_{1} \neq 0$. This means that the shock position is perturbed by $\mathcal{O}(\varepsilon)$, but the perturbation of the downstream solution is smaller. In this paper our goal is

$$
\mathbf{I}=-\alpha\left(x_{s}\right) x_{1}[\mathbf{v}] .
$$

Thus (2.16) is equivalent to requiring (see (A.9) and (A.10) in Appendix A)

$$
\mathbf{I}_{\mathbf{2}}:=\int_{-\infty}^{0} \alpha\left(x_{s}\right)\left(\mathbf{g}_{\mathbf{0}}(\tilde{x})-\mathbf{v}_{-}\right) d \tilde{x}+\int_{0}^{\infty} \alpha\left(x_{s}\right)\left(\mathbf{g}_{\mathbf{0}}(\tilde{x})-\mathbf{v}_{+}\right) d \tilde{x}=0 .
$$

One way of ensuring (2.17) is to require that the solution of (2.9) is of the form

$$
\mathbf{g}_{\mathbf{0}}(\tilde{x})=\mathbf{v}_{-}+\gamma(\tilde{x})[\mathbf{v}] .
$$

Here $\gamma$ should be a smooth, increasing function of $\tilde{x}$, antisymmetric around $(0,0.5)$, and with

$$
\gamma(-\infty)=0, \gamma^{\prime}(-\infty)=0, \gamma(\infty)=1, \gamma^{\prime}(\infty)=0 .
$$

Note that there is a one to one correspondence between $\mathbf{g}_{0}$ and $\gamma$. We also need to be able to express $\gamma^{\prime}$ in terms of $\gamma$ :

$$
\gamma^{\prime}=\psi(\gamma)
$$

Introduce the ansatz (2.18) into (2.10) and integrate, obtaining

$$
\mathbf{f}\left(\mathbf{v}_{-}+\gamma[\mathbf{v}]\right)-\mathbf{f}\left(\mathbf{v}_{-}\right)=\psi(\gamma) E[\mathbf{v}] .
$$

Clearly the ansatz is valid if the matrix $E$ is a projection to the subspace spanned by

$$
\mathbf{q}\left(\mathbf{g}_{0}\right):=\mathbf{f}\left(\mathbf{g}_{0}\right)-\mathbf{f}\left(\mathbf{v}_{-}\right),
$$

that is,

$$
E(\mathbf{g})=\frac{1}{\psi(\gamma)} \frac{\mathbf{q}\left(\mathbf{g}_{0}\right)\left(\mathbf{q}\left(\mathbf{g}_{0}\right)\right)^{T}}{\mathbf{q}\left(\mathbf{g}_{0}\right)^{T}[\mathbf{v}]}, \quad \gamma=\frac{\left(\mathbf{g}_{0}-\mathbf{v}_{-}\right)^{T}[\mathbf{v}]}{[\mathbf{v}]^{T}[\mathbf{v}]} .
$$

To ensure that $E$ is bounded when $\tilde{x} \rightarrow \pm \infty$ we require

$$
\lim _{\tilde{x} \rightarrow-\infty} \frac{\gamma(\tilde{x})}{\psi(\gamma(\tilde{x}))}=M_{-}, \quad \lim _{\tilde{x} \rightarrow \infty} \frac{\gamma(\tilde{x})-1}{\psi(\gamma(\tilde{x}))}=M_{+},
$$


where $\left|M_{-}\right|+\left|M_{+}\right|<\infty$. We have the following result directly from the following lemma.

Lemma 2.1. Assume $\left(\mathbf{q}\left(\mathbf{v}_{-}+y[\mathbf{v}]\right)\right)^{T}[\mathbf{v}]>0$ for all $0 \leq y \leq 1$, let $E$ be given by (2.22), and let $\gamma$ be a smooth, increasing function, antisymmetric around $(0,0.5)$ satisfying (2.19), (2.20), and (2.23). Then the inner problem (2.10) has a solution of the form (2.18).

Note that $E$ is positive semidefinite only. However, in the computations an $\varepsilon^{2} u_{x x}$ term is present, ensuring a positive definite viscosity matrix.

We now state the main result of this paper.

TheOREM 2.1. If the conditions of Lemma 2.1 are valid and Assumption 2.2 holds, then the error downstream of the shock is of order o( $\varepsilon)$.

Proof. If Lemma 2.1 and Assumption 2.2 hold, then the unique solution of (2.10) is of the form (2.18) and the integral $I$ in (2.14) is of the form (2.17). The system $(2.15)$ is solved by $\mathbf{w}_{1} \equiv 0$ and the result follows.

Remark 2.2. Theorem 2.1 states that the downstream error is of order $\mathrm{o}(\varepsilon)$. The calculations shown in section 4 suggest that the downstream error is merely of order $\mathcal{O}\left(\varepsilon^{2}\right)$. With a more elaborate matching argument, the analysis will also yield a downstream error of order $\mathcal{O}\left(\varepsilon^{2}\right)$.

3. Numerical implementation. In the previous section we derived an artificial viscosity matrix $E(\mathbf{g})$ given by $(2.22)$. In the computations we will use

$$
E\left(\mathbf{g}_{0}\right)=\frac{1}{\gamma_{0}^{\prime}(\tilde{x})} \frac{\mathbf{q}\left(\mathbf{g}_{0}\right) \mathbf{q}\left(\mathbf{g}_{0}\right)^{T}}{\mathbf{q}\left(\mathbf{g}_{0}\right)^{T}[\mathbf{v}]}, \quad \tilde{x}=\frac{x-x_{s}}{\varepsilon}
$$

with

$$
\mathbf{g}_{0}(\tilde{x})=\mathbf{v}_{-}+\gamma_{0}(\tilde{x})[\mathbf{v}], \quad \gamma_{0}(\tilde{x})=0.5(\tanh (\tilde{x})+1),
$$

and $\mathbf{q}\left(\mathbf{g}_{0}\right)$ is defined in (2.21). Note that $E\left(\mathbf{g}_{0}\right)$ is completely determined by $\mathbf{v}_{ \pm}, x_{s}$, and $\varepsilon$.

The expression (3.1) needs to be modified when used in a numerical computation. To begin with, both $\mathbf{q}$ and $\gamma_{0}^{\prime}$ tend to zero as $\tilde{x} \rightarrow \pm \infty$. However, for large $\tilde{x}$ we can linearize the expression for $\mathbf{q}$ around $\mathbf{v}_{ \pm}$and find

$$
\mathbf{q} \approx \begin{cases}\gamma_{0} J_{-}[\mathbf{v}] & \text { as } \tilde{x} \rightarrow-\infty \\ \left(\gamma_{0}-1\right) J_{+}[\mathbf{v}] & \text { as } \tilde{x} \rightarrow \infty\end{cases}
$$

Here $J_{ \pm}=J\left(\mathbf{v}_{ \pm}\right)$. By assumptions on $\gamma_{0}$, we finally find

$$
E\left(\mathbf{g}_{0}\right) \approx\left\{\begin{array}{l}
M_{-} \frac{J_{-}[\mathbf{v}][\mathbf{v}]^{T} J_{-}^{T}}{[\mathbf{v}]^{T} J_{-}^{T}[\mathbf{v}]} \\
M_{+} \frac{J_{+}[\mathbf{v}][\mathbf{v}]^{T} J_{+}^{T}}{[\mathbf{v}]^{T} J_{+}^{T}[\mathbf{v}]} \quad \text { as } \tilde{x} \rightarrow-\infty, \infty .
\end{array}\right.
$$

In a numerical computation the inviscid solution will in general not be known a priori. Hence $x_{s}$ and $\mathbf{v}_{ \pm}$must be approximated from the numerical solution. In this paper we consider only $\mathbf{v}_{ \pm}$as unknown. With approximate values for $\mathbf{v}_{ \pm}$, [f $]$will in general not equal zero. Thus

$$
\mathbf{q}_{-}:=\mathbf{f}-\mathbf{f}_{-} \neq \mathbf{f}-\mathbf{f}_{+}=: \mathbf{q}_{+} .
$$

We therefore define

$$
E_{ \pm}=\frac{1}{\gamma_{0}^{\prime}} \frac{\mathbf{q}_{ \pm} \mathbf{q}_{ \pm}^{T}}{\mathbf{q}_{ \pm}^{T}[\mathbf{v}]}
$$


By using the relation $[\mathbf{f}]=\mathbf{q}_{-}-\mathbf{q}_{+}$it is easy to see that

$$
\gamma_{0}^{\prime} E_{-}=\gamma_{0}^{\prime} E_{+}+\mathcal{O}([\mathbf{f}]) .
$$

That is, for small $[\mathbf{f}]$ and $\gamma_{0}^{\prime}$ sufficiently large, $E_{-} \approx E_{+}$.

In our computations we have used

$$
E\left(\mathbf{g}_{0}\right)= \begin{cases}M_{-} \frac{J_{-}[\mathbf{v}][\mathbf{v}]^{T} J_{-}^{T}}{[\mathbf{v}]^{T} J_{-}^{T}[\mathbf{v}]}, & \tilde{x}<-K, \\ \frac{1}{\gamma_{0}^{\prime}} \frac{\mathbf{q}_{-} \mathbf{q}_{-}^{T}}{\mathbf{q}_{-}^{T}[\mathbf{v}]}, & -K \leq \tilde{x}<0, \\ \frac{1}{\gamma_{0}^{\prime}} \frac{\mathbf{q}_{+} \mathbf{q}_{+}^{T}}{\mathbf{q}_{+}^{T}[\mathbf{v}]}, & 0 \leq \tilde{x}<K, \mathbf{v}, \\ M_{+} \frac{J_{+}[\mathbf{v}][\mathbf{v}]^{T} J_{+}^{T}}{[\mathbf{v}]^{T} J_{+}^{T}[\mathbf{v}]}, & \tilde{x}>K,\end{cases}
$$

where $\mathbf{q}_{ \pm}$are defined in (3.3), $J_{ \pm}=J\left(\mathbf{v}_{ \pm}\right)$and $M_{ \pm}$are defined in (2.23), and $K$ is a large constant.

Remark 3.1. If we use a first order approximation of the inviscid solution, we expect $[\mathbf{f}]=\mathcal{O}(h)$. This should yield $\mathcal{O}(h)$ perturbations of $E$ and $\mathbf{g}_{0}$. We expect this effect to be seen in $\mathbf{w}_{2}$ but not in $\mathbf{w}_{1}$.

Remark 3.2. In a fully automatic procedure, the shock location, $x_{s}$, has to be determined by the algorithm. This could be done, e.g., by looking at the eigenvalue that changes sign across the shock. As in the previous remark an $\mathcal{O}(h)$ error in the shock location is not seen in $\mathbf{w}_{1}$.

4. Numerical results. In this section the artificial viscosity matrix coefficient that was introduced in the previous section is tested in numerical calculations.

We solved (2.1) where

$$
\mathbf{v}=\left(\begin{array}{c}
\rho \\
u
\end{array}\right), \quad \mathbf{f}(\mathbf{v})=\left(\begin{array}{c}
\rho u \\
\frac{1}{2} u^{2}+\rho
\end{array}\right), \quad J=\frac{\partial \mathbf{f}}{\partial \mathbf{v}}=\left(\begin{array}{cc}
u & \rho \\
1 & u
\end{array}\right) .
$$

That is, the shallow water equations with a source term added. Here $\alpha(x)=\frac{1}{A} \frac{d A}{d x}$, and $A(x)=1.398+0.347 \tanh (5(x-0.4))$. The eigenvalues of the Jacobian $J$ are $\lambda_{1,2}=u \pm \sqrt{\rho}$ and the two Riemann invariants are $R_{1,2}=\frac{1}{2} u \pm \sqrt{\rho}$.

The boundary conditions were

$$
\begin{aligned}
\left(\begin{array}{c}
\rho \\
u
\end{array}\right) & =\left(\begin{array}{l}
\rho_{0} \\
u_{0}
\end{array}\right) \quad \text { at } x=0, \\
R_{2}(\rho, u) & =r \quad \text { at } x=1 .
\end{aligned}
$$

Here $\rho_{0}, u_{0}$, and $r$ are given boundary data.

We can explicitly calculate the inviscid solution

$$
\begin{gathered}
\rho(x) \equiv \rho_{0}, \quad u(x)=-\int_{0}^{x} \alpha\left(x^{\prime}\right) d x^{\prime} \quad \text { for } 0 \leq x<x_{s}, \\
\rho(x) \equiv \rho_{1}, \quad u(x)=-\int_{x}^{1} \alpha\left(x^{\prime}\right) d x^{\prime} \quad \text { for } x_{s}<x \leq 1 .
\end{gathered}
$$

Here $x_{s}$ and $\rho_{1}$ are determined by the Rankine-Hugoniot condition

$$
[\mathbf{f}]=0 \quad \text { at } x=x_{s},
$$

where $\mathbf{f}$ is defined in (4.1). 
We used the semidiscrete scheme

$$
\left(\mathbf{v}_{i}\right)_{t}+D_{0} \mathbf{f}\left(\mathbf{v}_{i}\right)+\alpha\left(x_{i}\right) \mathbf{v}_{i}=h D_{+} E_{i} \phi_{i} D_{-} \mathbf{v}_{i}+h^{2} \varepsilon_{0} D_{+} D_{-} \mathbf{v}_{i} .
$$

Here $x_{i}=i h, h=1 / N, i=0,1, \ldots, N$, and $\mathbf{v}_{i}$ is a grid function, where $\mathbf{v}_{i} \approx \mathbf{v}\left(x_{i}\right)$. Also, $\phi_{i}=\phi\left(x_{i}\right)$.

The operators $D_{0}$ and $D_{ \pm}$are defined as

$$
D_{0} u_{i}=\left(u_{i+1}-u_{i-1}\right) / 2 h, \quad D_{+} u_{i}=\left(u_{i+1}-u_{i}\right) / h, \quad D_{-} u_{i}=\left(u_{i}-u_{i-1}\right) / h .
$$

The artificial viscosity term $E_{i}$ is of the form (3.4) and was computed in the following way. First, the shock position of the inviscid solution, $x_{s}$, was considered as known. Second, the $\tilde{x}$-space was discretized as $\tilde{x}_{j}=\tilde{h}(j-N / 2)$, where $j=$ $0,1, \ldots, \tilde{N}, \tilde{h}=15 / \tilde{N}$. Next, the matrix $E\left(\mathbf{g}_{0}\left(\tilde{x}_{j}\right)\right)$ was evaluated for $j=0,1, \ldots, \tilde{N}$. Finally, the $\tilde{x}$-space was mapped onto the interval $\left\{x: x_{m}^{-} \leq x-x_{s} \leq x_{m}^{+}\right\}$in $x$ space. The $E_{i}$ used in (4.6) was taken as the $E\left(\mathbf{g}_{0}\left(\tilde{x}_{j}\right)\right)$ for which $\min _{j}\left|x_{i}-h \tilde{x}_{j}\right|$ was obtained for the corresponding $\tilde{x}_{j}$. The number of grid points in the inner solution was $\tilde{N}=100$ in all our calculations shown in this report. An increase of $\tilde{N}$ to $\tilde{N}=200$ did not alter the results. Also, in all our calculations $\varepsilon_{0}=30$.

The switch $\phi$ is given by (see [4])

$$
\phi(x)=\left\{\begin{array}{cc}
\left.0.5 \tanh \left(\left(x-x_{s}-5 \tau h\right) / \tau h\right)+0.5\right), & x \leq x_{s} \\
\left.-0.5 \tanh \left(\left(x-x_{s}-5 \tau h\right) / \tau h\right)+0.5\right), & x>x_{s} .
\end{array}\right.
$$

In (4.7), $\tau$ is a parameter which alters the steepness and the locations of the large gradients of the function $\phi$. In all calculations presented below $\tau=1.1$. This means that the large viscosity $h E_{i}$ dominates in the region close to the shock layer, while the smaller viscosity $h^{2} \varepsilon_{0}$ dominates elsewhere.

We used the following boundary conditions:

$$
\begin{array}{ll}
v_{0}^{(1)}=\rho_{0}, & v_{0}^{(2)}=u_{0}, \\
\left(R_{1}\right)_{N}=2\left(R_{1}\right)_{N-1}-\left(R_{1}\right)_{N-2}, & \left(R_{2}\right)_{N}=r,
\end{array}
$$

where $v_{0}^{(1,2)}$ are the components of $\mathbf{v}_{0}$ and $R_{1,2}$ are the two Riemann invariants.

The system of ODEs (4.6) was integrated with the classical fourth order RungeKutta method until the residual was of order $10^{-12}$. The time step was $1 \cdot 10^{-4}$. All calculations were performed in double precision.

The order of accuracy was estimated in the standard way by calculating the steady state solution of (4.6) for $N=100,200,400,800$, and 1600 and then computing

$$
p_{i}=\log \left(\frac{u_{N}\left(x_{i}\right)-u^{i n v}\left(x_{i}\right)}{u_{2 N}\left(x_{i}\right)-u^{i n v}\left(x_{i}\right)}\right) / \log (2) .
$$

Here $p$ is the order of accuracy, $u_{N}$ the first component of $\mathbf{v}_{i}$ in the solution of (4.6), and $u^{i n v}$ the corresponding inviscid solution (4.4), respectively. All results were qualitatively the same when $p$ was calculated from $\rho$ and $u$, respectively.

As initial data we used the steady state solution of (4.6) with $E_{i}=h \mu I$, where $I$ is the identity matrix. Here $\mu$ was chosen such that the shock layer was well resolved. With this choice of viscosity coefficient the order of accuracy was 2 upstream of the shock and 1 downstream, respectively (see Figure 4.1).

Two different types of approximations of $v_{ \pm}$in the evaluation of the viscosity matrix $E$ were examined. First, we used the inviscid solution at the shock position 


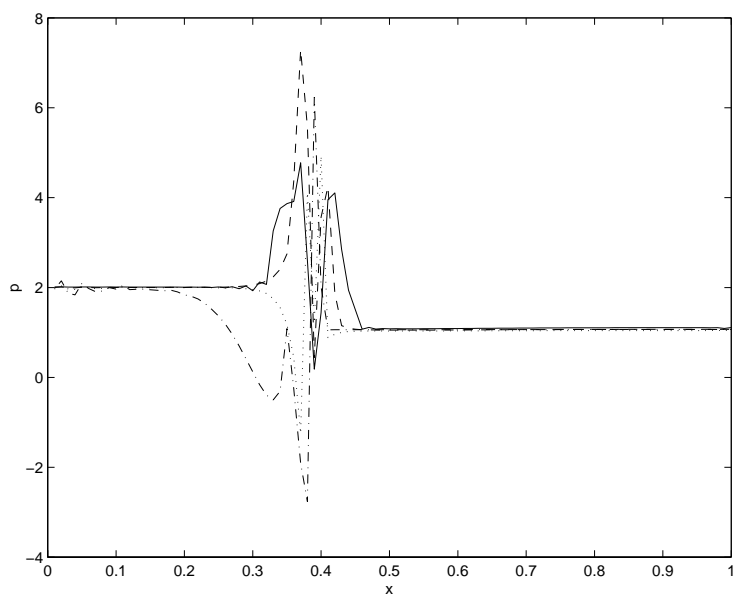

FIG. 4.1. Order of accuracy computed from (4.8) of the initial data. Solid line: $N=100$, dashed line: $N=200$, dotted line: $N=400$, dash-dotted line: $N=800$.

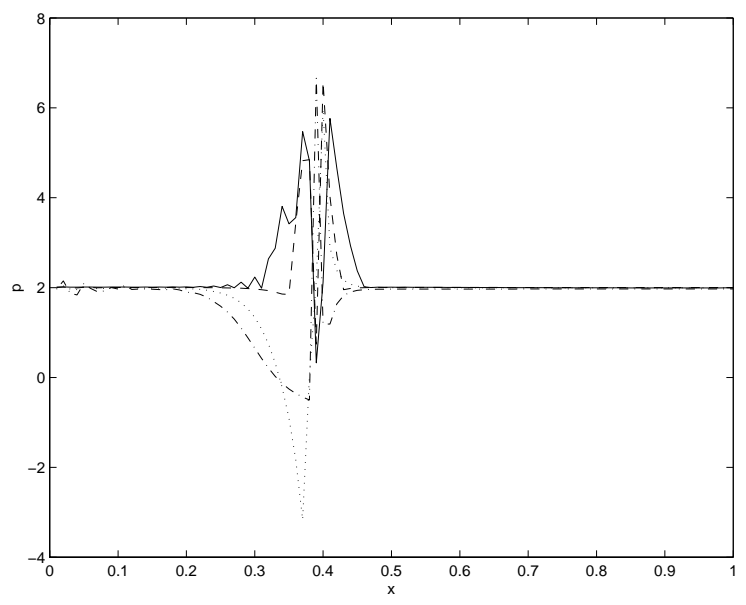

FIG. 4.2. Order of accuracy computed from (4.8). The values of $\mathbf{v}_{ \pm}$in (3.4) were the true inviscid solution. Solid line: $N=100$, dashed line: $N=200$, dotted line: $N=400$, dash-dotted line: $N=800$

$x_{s}$ as boundary data. Second, we used the data from the solution close to the shock layer. The data $v_{ \pm}$was hence updated in every time step and differed for every grid.

In Figure 4.2 we show the order of accuracy when $v_{ \pm}$in (3.4) was the true inviscid solution at the shock position $x_{s}$. We see that the order of accuracy has increased from 1 in the initial data to 2 , downstream of the shock.

To simulate a more realistic computation, we let $\mathbf{v}_{ \pm}$in (3.4) be the numerical solution at $x_{m}^{ \pm}$. Here $x_{m}^{-}$was taken as the first $x$-value for which $\phi(x)>\delta$. Accordingly, $x_{m}^{+}$was the last $x$-value for which $\phi(x)>\delta$. Here $\delta$ is a small number and $\phi$ is defined in (4.7). We used $\delta=0.05$ in all calculations presented in this article. The results 


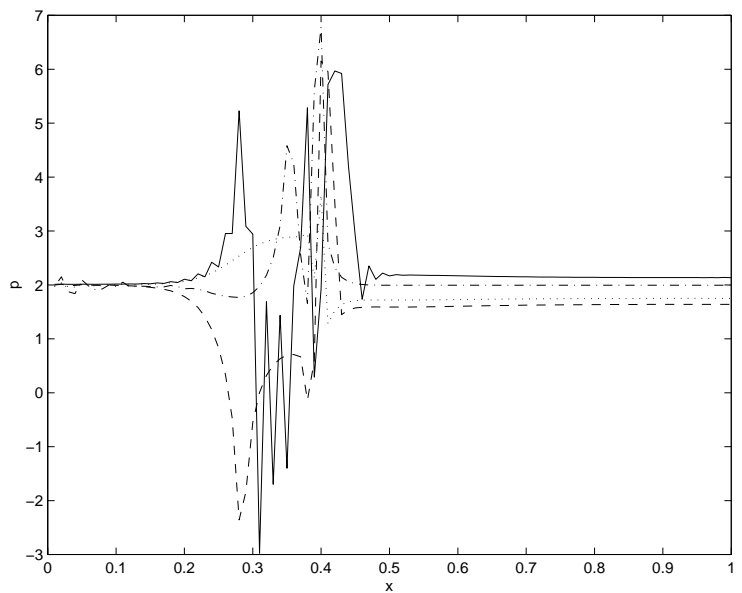

FIG. 4.3. Order of accuracy computed from (4.8). The values of $\mathbf{v}_{ \pm}$in (3.4) were approximated by data close to the shock from the numerical solution in each time step. Solid line: $N=100$, dashed line: $N=200$, dotted line: $N=400$, dash-dotted line: $N=800$.
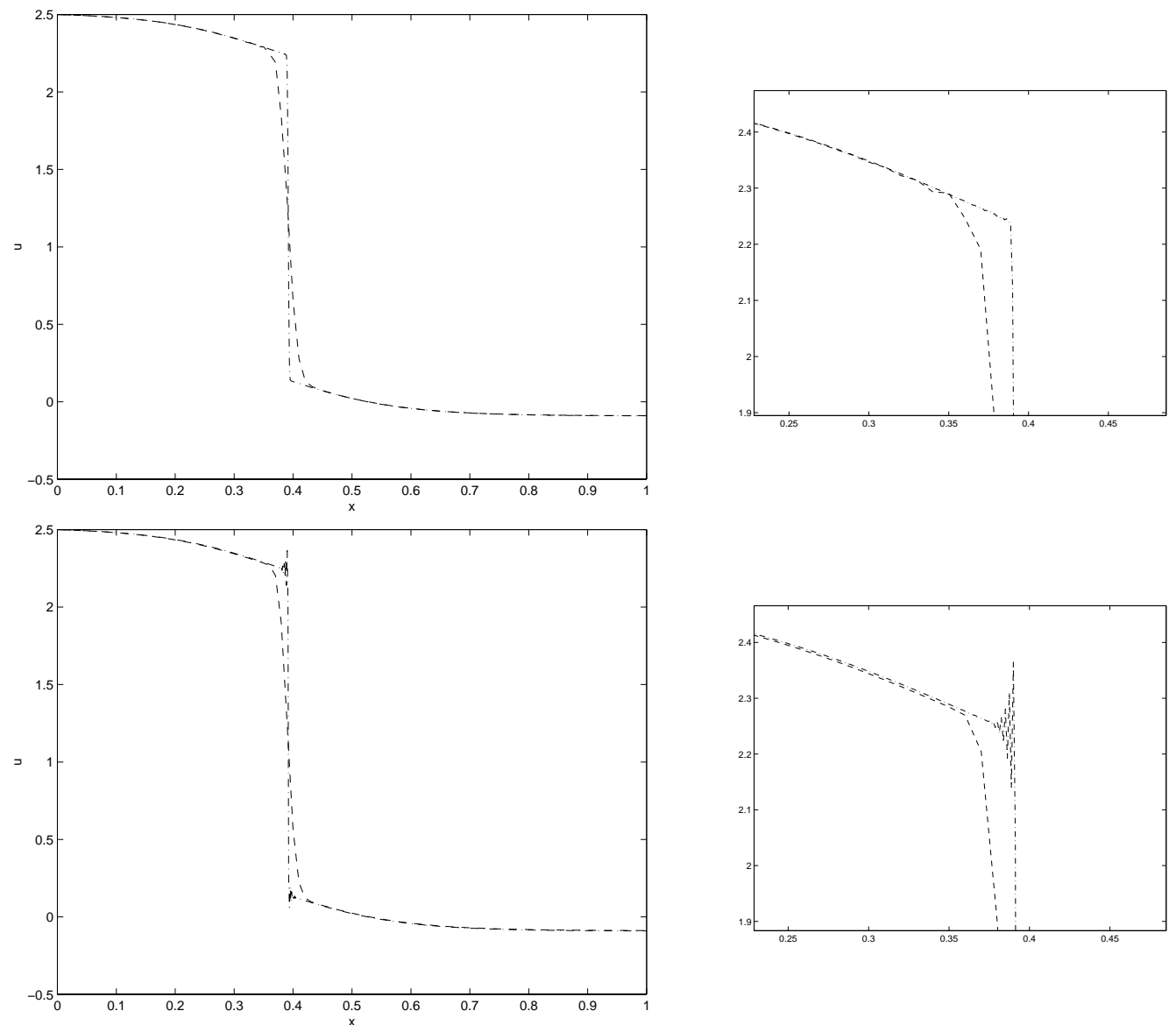

FIG. 4.4. Numerical solutions of (2.1) using (4.6) in space with $E$ chosen as in (3.4) and $\varepsilon_{0}=30$ (upper) and $E=0$ and $\varepsilon_{0}=100$ (lower). Dashed line: $N=100$, dash-dotted line: $N=800 . A$ blow up of the region upstream of the shock is shown to the right. 
were insensitive to the choice of $\delta$. Here data was updated in every time step and it was different for different grid sizes. The order of accuracy can be seen in Figure 4.3.

The aim of introducing an artificial viscosity term of $\mathcal{O}(h)$ is to reduce oscillations at the shock and stabilize the calculations. We therefore found it interesting to compare solutions obtained with our new artificial viscosity term and those obtained with a viscosity term of $\mathcal{O}\left(h^{2}\right)$. Hence, both solutions are second order away from the shock. The two different solutions are shown in Figure 4.4 for two different grid sizes. In the calculation with the viscosity term of $\mathcal{O}\left(h^{2}\right)$ we used $\varepsilon_{0}=100$ in the whole domain. Figure 4.4 shows that as the grid was refined, as expected, oscillations appeared with the smaller amount of artificial viscosity, while with the artificial viscosity introduced in this paper the solution stayed smooth.

5. Conclusions and future directions. Using a matched asymptotic expansion analysis we have analyzed the reduction of higher order accurate methods in space to first order downstream of shocks. By a detailed analysis of the inner problem and a subsequent modification of the artificial viscosity, the first order error downstream of the shock is removed. In the inner region, the model problem that is analyzed here can be motivated only for weak shocks. However, we present numerical calculations where the order of accuracy was approximately 2 downstream of a nonweak shock. In future work, it would be interesting to see if the procedure can be continued to reduce the downstream error to be of even higher order in $\varepsilon$.

Appendix A. In this section we derive, for completeness, (2.14), that is, the boundary conditions for the first order perturbation of the viscous solution, $\mathbf{w}_{1}$, at the matching point $x_{m}^{+}$. The derivations follow the analysis presented in [4].

In the following, $\mathbf{v}$ and $\mathbf{u}$ denote the steady solution of the inviscid, (2.1), and viscous, (2.5), problem, respectively. Also, $x_{s}$ is the shock position.

Integration of the viscous equation (2.5) over the shock layer, from the matching point $x_{m}^{-}$to the matching point $x_{m}^{+}$, yields

$$
[\mathbf{f}(\mathbf{u})]_{x_{m}^{-}}^{x_{m}^{+}}+\int_{x_{m}^{-}}^{x_{m}^{+}} \alpha(x) \mathbf{u} d x=\varepsilon^{2}\left[\mathbf{u}_{x}\right]_{x_{m}^{-}}^{x_{m}^{+}} .
$$

Here $x_{m}^{-}$and $x_{m}^{+}$are defined in Assumption 2.2. We will introduce the expansions (2.7) into this equality and consider terms of $\mathcal{O}(1)$ and $\mathcal{O}(\varepsilon)$. At the matching points we use the outer solution and in the shock layer the inner solution.

As expected to zeroth order in $\varepsilon$, (A.1) is equivalent to the Rankine-Hugoniot relation $(2.2)$. This is true since

$$
[\mathbf{f}(\mathbf{u})]_{x_{m}^{-}}^{x_{m}^{+}}=[\mathbf{f}(\mathbf{v})]_{x_{m}^{-}}^{x_{m}^{+}}+\mathcal{O}(\varepsilon)=[\mathbf{f}(\mathbf{v})]_{x_{s}^{-}}^{x_{s}^{+}}+\mathcal{O}(M),
$$

and the other terms in (A.1) are of higher order in $\varepsilon$. Here $M$, introduced in Assumption 2.2, has the properties $\varepsilon \ll M \ll 1$, and $M \rightarrow 0$ as $\varepsilon \rightarrow 0$.

To the next order we have

$$
[\mathbf{f}(\mathbf{u})]_{x_{m}^{-}}^{x_{m}^{+}}=[\mathbf{f}(\mathbf{v})]_{x_{m}^{-}}^{x_{m}^{+}}+\varepsilon\left[J(\mathbf{v}) \mathbf{w}_{1}\right]_{x_{m}^{-}}^{x_{m}^{+}}+\mathcal{O}\left(\varepsilon^{2}\right) .
$$

By integrating the steady inviscid equation over the same interval, we obtain

$$
[\mathbf{f}(\mathbf{v})]_{x_{m}^{-}}^{x_{m}^{+}}=-\int_{x_{m}^{-}}^{x_{m}^{+}} \alpha(x) \mathbf{v} d x
$$


After taking into account that $\mathbf{w}_{1} \equiv 0$ to the left of the shock layer, and introducing (A.2) and (A.3) into (A.1), we find

$$
\varepsilon J\left(\mathbf{v}\left(x_{m}^{+}\right)\right) \mathbf{w}_{1}\left(x_{m}^{+}\right)+\alpha\left(x_{s}\right) \int_{x_{m}^{-}}^{x_{m}^{+}}(\mathbf{u}-\mathbf{v}) d x=\mathcal{O}\left(\varepsilon^{2}\right) .
$$

Denote the integral

$$
\mathbf{I}_{1}:=\alpha\left(x_{s}\right) \int_{x_{m}^{-}}^{x_{m}^{+}}(\mathbf{u}-\mathbf{v}) d x
$$

We will now express $\mathbf{I}_{1}$ by using the first term of the inviscid solution at $x=x_{s}$, the inner solution, and $x_{1}$, respectively.

In order to obtain the equation for the inner solution we introduce the stretched variable $\tilde{x}=\left(x-x_{s}\right) / \varepsilon$ into $(2.5)$, yielding

$$
\mathbf{f}(\mathbf{u})_{\tilde{x}}+\varepsilon \alpha\left(\varepsilon \tilde{x}+x_{s}\right) \mathbf{u}=\left(\phi(\tilde{x}) E \mathbf{u}_{\tilde{x}}\right)_{\tilde{x}}+\varepsilon \mathbf{u}_{\tilde{x} \tilde{x}} .
$$

Thus the first term in the inner expansion satisfies

$$
\mathbf{f}\left(\mathbf{g}_{0}\right)_{\tilde{x}}=\left(\phi(\tilde{x}) E \mathbf{g}_{0 \tilde{x}}\right)_{\tilde{x}} .
$$

The first order perturbation of the position of the shock layer is $x_{1}$. Clearly, $x_{1}$ cannot be determined by (2.9). Let $\mathbf{g}(\tilde{x})$ satisfy

$$
\mathbf{f}(\mathbf{g})_{\tilde{x}}=\left(E \mathbf{g}_{\tilde{x}}\right)_{\tilde{x}},
$$

together with the additional condition that the smallest eigenvalue of $J(\mathbf{g})$ vanishes at $\tilde{x}=0$. Here $J$ is the Jacobian of the flux function $\mathbf{f}$. Then, for sufficiently large $K$, $\mathbf{g}_{0}(\tilde{x})=\mathbf{g}\left(\tilde{x}-x_{1}\right)$, except for exponentially small terms, where $K$ is defined in (2.6). Below we will determine $x_{1}$ by considering the outer problem to $\mathcal{O}(\varepsilon)$.

The integral $\mathbf{I}_{1}$ becomes with the inner variable $\tilde{x}=\left(x-x_{s}\right) / \varepsilon$,

$$
\mathbf{I}_{1}=\varepsilon \alpha\left(x_{s}\right) \int_{\tilde{x}_{m}^{-}}^{\tilde{x}_{m}^{+}}(\mathbf{u}-\mathbf{v}) d \tilde{x} .
$$

Here $\tilde{x}_{m}^{+}=\left(x_{m}^{+}-x_{s}\right) / \varepsilon$ and $\tilde{x}_{m}^{-}=\left(x_{m}^{-}-x_{s}\right) / \varepsilon$. Introduce the asymptotic expansion of the inner solution

$$
\begin{array}{r}
\left.\mathbf{I}_{1}=\varepsilon \alpha\left(x_{s}\right) \int_{\tilde{x}_{m}^{-}}^{\tilde{x}_{m}^{+}}\left(\mathbf{g}_{0}(\tilde{x})+\varepsilon \mathbf{g}_{1}(\tilde{x})+\ldots\right)-\mathbf{v}\left(\varepsilon \tilde{x}+x_{s}\right)\right) d \tilde{x} \\
=\varepsilon \alpha\left(x_{s}\right) \int_{-\infty}^{0}\left(\mathbf{g}_{0}(\tilde{x})-\mathbf{v}_{-}\right) d \tilde{x}+\varepsilon \alpha\left(x_{s}\right) \int_{0}^{\infty}\left(\mathbf{g}_{0}(\tilde{x})-\mathbf{v}_{+}\right) d \tilde{x}+\boldsymbol{o}(\varepsilon) \\
:=\varepsilon \mathbf{I}_{2}+\boldsymbol{o}(\varepsilon) .
\end{array}
$$

Here $\mathbf{v}_{ \pm}$is the inviscid solution of the left and the right branches, respectively, at the inviscid shock position $x_{s}$. Since $\mathbf{g}_{0}(\tilde{x})=\mathbf{g}\left(\tilde{x}-x_{1}\right)$ we have that

$$
\begin{array}{r}
\mathbf{I}_{2}=\alpha\left(x_{s}\right) \int_{-\infty}^{0}\left(\mathbf{g}(\tilde{x})-\mathbf{v}_{-}\right) d \tilde{x} \\
+\alpha\left(x_{s}\right) \int_{0}^{\infty}\left(\mathbf{g}(\tilde{x})-\mathbf{v}_{+}\right) d \tilde{x}+x_{1} \alpha\left(x_{s}\right)[\mathbf{v}] \\
:=\mathbf{I}_{3}+x_{1} \alpha\left(x_{s}\right)[\mathbf{v}] .
\end{array}
$$


Finally, with (A.9) and (A.10) in (A.4) and noting that

$$
J^{+} \equiv J\left(\mathbf{v}_{+}\right)=J\left(\mathbf{v}\left(x_{m}^{+}\right)\right)+\mathcal{O}(M),
$$

we see that the boundary condition for $\mathbf{w}_{1}$ at $x=x_{m}^{+}$is

$$
\left.J^{+} \mathbf{w}_{1}\left(x_{m}^{+}\right)+x_{1} \alpha\left(x_{s}\right)[\mathbf{v}]\right)=-\mathbf{I}_{3} .
$$

\section{REFERENCES}

[1] M. H. Carpenter And J. H. Casper, The accuracy of shock capturing in two spatial dimensions, in Proceedings of the 13th AIAA Computational Fluid Dynamics Conference, Snowmass Village, CO, Part 1, AIAA paper 97-2107, 1997, pp. 488-498.

[2] J. Casper And M. H. CARPenter, Computational considerations for the simulation of shockinduced sound, SIAM J. Sci. Comput., 19 (1998), pp. 813-828.

[3] R. L. Clark and K. D. Frampton, Aeroelastic structural acoustic coupling: Implications on the control of turbulent boundary-layer noise transmission, J. Acoust. Soc. Amer., 102 (1997), pp. 1639-1647.

[4] G. Efraimsson and G. Kreiss, A remark on numerical errors downstream of slightly viscous shocks, SIAM J. Numer. Anal., 36 (1999), pp. 853-863.

[5] B. Engquist And B. SJögreen, The convergence rate of finite difference schemes in the presence of shocks, SIAM J. Numer. Anal., 35 (1998), pp. 2464-2485.

[6] H. Jurgens And D. ZING, Implementation of a high-accuracy finite-difference scheme for linear wave phenomena, in Proceedings from the International Conference on Spectral and Higher Order Methods 95, paper 16, Houston, TX, 1995.

[7] S. Karni And S. Čanić, Computations of slowly moving shocks, J. Comput. Phys., 136 (1997), pp. 132-139.

[8] J. Kevorkian and J. D. Cole, Perturbation Methods in Applied Mathematics, SpringerVerlag, New York, Berlin, 1981.

[9] H.-O. Kreiss and J. Lorenz, Initial-Boundary Value Problems and the Navier-Stokes Equations, Academic Press, Boston, 1989.

[10] H.O. Kreiss And J. Oliger, Comparison of accurate methods for the integration of hyperbolic equations, Tellus, 24 (1972), pp. 199-215.

[11] P. A. Lagerstrom, Matched Asymptotic Expansions, Springer-Verlag, New York, Berlin, 1988.

[12] P. D. LAx, Hyperbolic Systems of Conservation Laws and the Mathematical Theory of Shock Waves, CBMS-NSF Regional Conf. Ser. in Appl. Math. 11, SIAM, Philadelphia, 1973.

[13] R. J. LeVeque, Numerical Methods for Conservations Laws, Birkhäuser, Verlag, Basel, 1990.

[14] D.-L. LIU AND R. C. WAAG, Harmonic amplitude distribution in a wideband ultrasonic wavefront after propagation through human abdominal wall and breast specimens, J. Acoust. Soc. Amer., 101 (1997), pp. 1172-1183.

[15] M. LOU AND J. A. RIAL, Characterization of geothermal reservoir crack patterns using shearwave splitting, Geophysics, 62 (1997), pp. 487-494.

[16] M. OKoniewski And M. A. StuChly, A study of the handset antenna and human body interaction, IEEE Trans. Microwave Theory Tech., 44 (1996), pp. 1855-1864.

[17] C. Pruett, T. Zang, C. Chang, and M. CARPenter, Spatial direct numerical simulation of high speed boundary-layer flows, Part I: Algorithmic considerations and validation, Theor. Comput. Fluid Dyn., 7 (1995), pp. 397-424.

[18] A. TAFLOVE, Re-inventing electromagnetic supercomputing solution of Maxwell's equations via direct time integration on space grid, 30th AIAA Aerospace Sciences Meeting, Reno, NV, AIAA paper 92-0333, 1992.

[19] C. Tam and J. WebB, Dispersion-relation-preserving finite difference schemes for computational acoustics, J. Comput. Phys., 107 (1993), pp. 262-281.

[20] C. TAM, Computational aeroacoustics: Issues and methods, AIAA J., 33 (1995), pp. 1788-1796. 